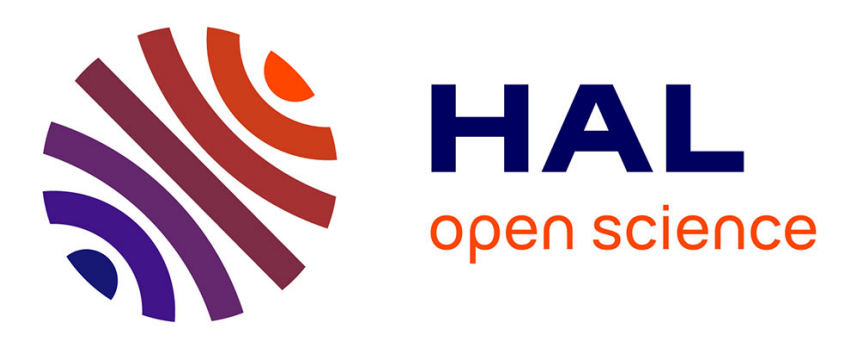

\title{
Continuous Growth of Germanene and Stanene Lateral Heterostructures
}

Tsuyoshi Ogikubo, Hiroki Shimazu, Yuya Fujii, Koichi Ito, Akio Ohta, Masaaki Araidai, Masashi Kurosawa, Guy Le Lay, Junji Yuhara

\section{- To cite this version:}

Tsuyoshi Ogikubo, Hiroki Shimazu, Yuya Fujii, Koichi Ito, Akio Ohta, et al.. Continuous Growth of Germanene and Stanene Lateral Heterostructures. Advanced Materials Interfaces, 2020, 7 (10), pp.1902132. 10.1002/admi.201902132 . hal-03202625

\section{HAL Id: hal-03202625 \\ https://hal.science/hal-03202625}

Submitted on 20 Apr 2021

HAL is a multi-disciplinary open access archive for the deposit and dissemination of scientific research documents, whether they are published or not. The documents may come from teaching and research institutions in France or abroad, or from public or private research centers.
L'archive ouverte pluridisciplinaire HAL, est destinée au dépôt et à la diffusion de documents scientifiques de niveau recherche, publiés ou non, émanant des établissements d'enseignement et de recherche français ou étrangers, des laboratoires publics ou privés. 


\title{
Continuous Growth of Germanene and Stanene Lateral Heterostructures
}

\author{
Tsuyoshi Ogikubo, Hiroki Shimazu, Yuya Fujii, Koichi Ito, Akio Ohta, Masaaki Araidai, \\ Masashi Kurosawa, Guy Le Lay, and Junji Yuhara
}

\begin{abstract}
Group 14 elemental post-graphene materials receive much attention because of their outstanding properties, typically, as robust 2D topological insulators. Their heterostructures are a main target in view of disruptive applications. Here, the realization of striking in-plane lateral heterostructures between germanene and stanene are shown, which are sustainable 2D Ge- and Snbased graphene analogs, but with a strong intrinsic spin-orbit coupling. A unique combination of atomic segregation epitaxy (ASE) and molecular beam epitaxy (MBE) for the in situ continuous fabrication of nearly atomically precise lateral multijunction heterostructures, respectively, consisting of atom-thin germanene and stanene on a $\mathrm{Ag}(111)$ thin film is used. Scanning tunneling microscopy (STM) observations at atomic scale and low-energy electron diffraction
\end{abstract}

testify that germanene and stanene sheets without intermixing are prepared simultaneously on the same terraces at wide scale: tin and germanium atoms neither exchange their sites nor adsorb on the germanene and stanene sheets. The atomic structure of the boundary between germanene and stanene

is derived from STM images, while scanning tunneling spectroscopy reveals key electronic features at the heterojunction. This innovative synergetic approach of ASE and MBE growths offers great flexibility for the realization of unprecedented lateral 2D heterostructures.

\section{Introduction}

Group 14 elemental 2D materials with honeycomb struc- ture beyond graphene have attracted considerable interest recently. At variance with graphene, for which the opening of a band gap in its electronic structure is difficult, these novel 2D materials are expected to possess sizeable band gaps, making them typically suitable for elec- tronic applications. Furthermore, theory predicts that they are robust topological insulators, even up to about room tem- perature (RT) and above for germanene and stanene.

In experiment, these artificial 2D materials were firstly realized in situ by molecular beam epitaxy (MBE) for silicene in 2012,[1,2] germanene in 2014,[3] stanene in 2015, [4] and finally plumbene in 2019.[5] One of the next targets is the growth of in-plane heterostructures because abrupt lateral interfaces promise controlled heterojunction functionali- ties. Lateral heterostructures of graphene and hexagonal boron nitride $(\mathrm{h}-\mathrm{BN})$ were grown on $\mathrm{Cu}(110)$ in 2014.[6] First-princi- ples calculations on the electronic proper- ties of graphene quantum dots embedded in monolayer h-BN sheets typically indi- cate that the h-BN band gap shrinks upon

increasing the diameter of the graphene dots.[7] Besides lat- eral graphene/h-BN heterostructures, fabrication of several in-plane heterostructures of transition metal dichalcogenides has also been reported. [8] Very recently, vertical heterostruc- tures have been 
prepared by boron intercalation underneath graphene, while, more strikingly, lateral integration of boro- phene with graphene has been realized.[9] At variance, there is no experimental report of the realization of such monolayer in-plane heterostructures for the group 14 post-graphene 2D materials, although each of them typically possesses very high hole and electron mobilities.[10] As stressed in Ref. [9], compared to vertical junctions with essentially van der Waals bonding, generally fabricated by mechanical stacking, 2D lat- eral heterostructures with covalent lateral stitching require bottom-up synthesis, which explains why such realizations are rare. However, the covalent in-plane bonding of lateral heterointerfaces for imperfect lattice matching often results in wide interfaces, where disorder and intermixing can reduce the structural and electronic abruptness. As a consequence, finding appropriate 2D materials and identifying synthetic conditions that yield nearly atomically sharp heterostructures is of major interest.

The energy gaps for silicene, germanene, and stanene are not so large, ranging from 0.002 to 0.03 , and $0.1 \mathrm{eV}$, respectively.[11] Yet, 2D pseudomorphic heterostructures, for example, of silicene and germanene ribbons, might be used to control the electronic band structures, because the band gaps are made tunable by adjusting the widths of the respective nanoribbons.[12,13] Nevertheless, the realization of post-graphene lateral heterostructures based on group 14 elements, which is crucial for the future fabrication of heterojunctionembedded 2D devices compatible with conventional Si-based technology, remains a daunting challenge.

Silicene has been initially synthesized in situ on $\mathrm{Ag}(111), \mathrm{ZrB}_{2}(0001)$, and $\operatorname{Ir}(111)[1,2,14]$ Next, the growth of germanene by MBE has been reported on $\mathrm{Au}(111),[3] \mathrm{Pt}(111),[15] \mathrm{Al}(111),[16]$ $\mathrm{Sb}(111),{ }^{[17]} \mathrm{Cu}(111),{ }^{[18]} \mathrm{Ag}(111),{ }^{[19]} \mathrm{Ge}(110),{ }^{[20]} \mathrm{MoS}_{2},{ }^{[21]}$ graphite, [22] and $\mathrm{Ge}(111)$.[23] Recently, germanene has been also prepared on $\mathrm{Ag}(111)$ thin films by atomic segregation epitaxy (ASE).[24] For the MBE growth of stanene, Bi2Te3(111), [4] Sb(111), [25] Ag(111), [26] and $\mathrm{Cu}(111)^{[27]}$ substrates have been adopted. Up to now, the $\mathrm{Ag}(111)$ surface appears as the most promising template for the heterostructure growth of all group 14 post-graphene materials. According to the phase diagram, Ge and Sn are immiscible at any ratio,[28] while $\mathrm{Ge}$ and $\mathrm{Si}$ can make all proportional solid solutions.[29] Therefore, germanene and stanene appear as a promising combination for realizing a lateral heterostructure on $\mathrm{Ag}(111)$.

In this work, we achieved lateral in-plane heterostructures of monolayer germanene and stanene on a silver (111) thin film epitaxially grown on a $\mathrm{Ge}(111)$ substrate, as shown in Figure 1. With a synergetic combination of in situ ASE for germanene and MBE for stanene, we found the appropriate kinetic conditions to realize simultaneously their lateral growths. Typically, after initial annealing of the sample at $500{ }^{\circ} \mathrm{C}$, during slow cooling Ge segregates at the top surface of the $\mathrm{Ag}(111)$ thin film, which favors $2 \mathrm{D}$ germanene islanding at about $200{ }^{\circ} \mathrm{C}$, the temperature where we per- formed the simultaneous deposition of 0.3 monolayers of Sn. By this procedure we obtained remarkably precise ger- manene/stanene heterojunctions without significant replace- ment of $\mathrm{Ge}$ atoms in stanene and vice versa. In experiments, the atomic and electronic structures have been determined by low-energy electron diffraction (LEED), scanning tun- neling microscopy (STM), and scanning tunneling spectros- copy (STS). 


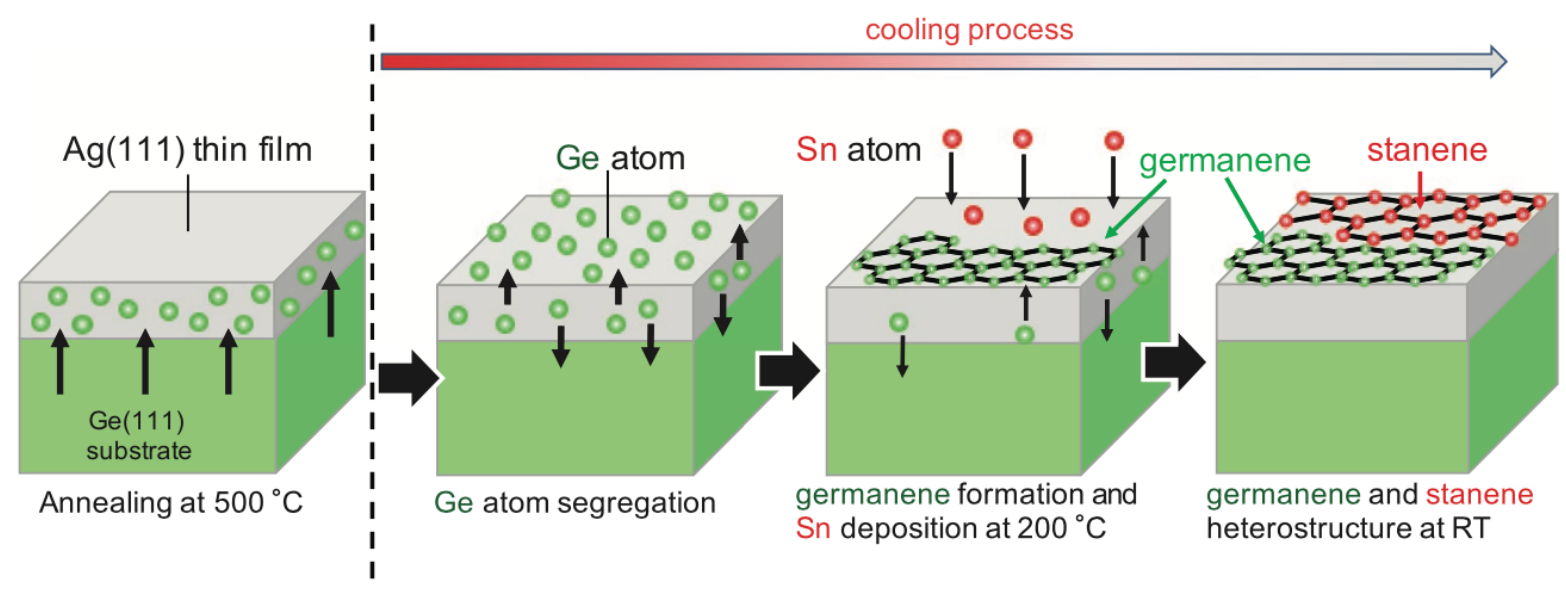

Figure 1. Illustration of the sequential formation of lateral heterostructures of germanene and stanene, prepared on a $\mathrm{Ag}(111)$ thin film grown on a $\mathrm{Ge}(111)$ substrate after annealing at 500 ${ }^{\circ} \mathrm{C}$, followed by slow cooling. A Sn deposition is performed at $200^{\circ} \mathrm{C}$ during the Ge segregation and germanene formation processes while cooling, resulting in the growth of lateral heterostructures of germanene and stanene observed at room temperature.

\section{Results and Discussion}

Figure 2a displays the LEED pattern for a stanene sheet formed at $200{ }^{\circ} \mathrm{C}$ by MBE over a $\mathrm{Ag}_{2} \mathrm{Sn}$ initial surface alloy grown on a $\mathrm{Ag}(111)$ single crystal, in conditions sim- ilar to those used in our previous work on the synthesis of stanene.[26] It shows the primitive spots of epitaxial stanene with a unit cell $0.50 \mathrm{~nm}$ in size (see Figure $2 \mathrm{e}$ ), corresponding to a $\mathrm{Ag}(111)(\sqrt{3}$ $\times \sqrt{3}) \mathrm{R} 30^{\circ}$ super periodicity. The corresponding STM image, revealing the honeycomb atomic arrangement of epitaxial monolayer stanene having a $0.50 \mathrm{~nm}$ unit cell, in perfect match with the underlying $\mathrm{Ag}_{2} \mathrm{Sn}$ surface alloy with $(\sqrt{3} \times \sqrt{3}) \mathrm{R} 30^{\circ}$ superstructure with respect to $\mathrm{Ag}(111)$, is displayed in Figure $2 \mathrm{~b}$. Figure $2 \mathrm{c}$ shows the LEED pattern for a complete germanene sheet formed also at $200{ }^{\circ} \mathrm{C}$ on top of a $\mathrm{Ag}(111)$ thin film previously grown on $\mathrm{Ge}(111)$ upon segregation of germanium during slow cooling, after initial heating of the specimen at $500^{\circ} \mathrm{C}$. The primitive spots originating from the honeycomb lattice of germanene, which correspond to $\mathrm{Ag}(111)$ "1.35 × 1.35"R30 ${ }^{\circ}$ with $0.39 \mathrm{~nm}$ size (see Figure $2 \mathrm{e}$ ), are clearly observed. Additional spots originate from the prominent characteristic $2 \mathrm{D}(3 \sqrt{2} 2 \times 3 \sqrt{21}) \mathrm{R} 10.9^{\circ}$ germanene superstructure observed in STM imaging (shown in Figure 2d), matching a $\mathrm{Ag}(111)(7 \sqrt{7} \times$ $7 \sqrt{7}) \mathrm{R} 19^{\circ}$ superstructure. Here, we emphasize that these LEED pat- terns and STM images are in perfect accordance with those obtained in previous papers. [24,26] 
(a)

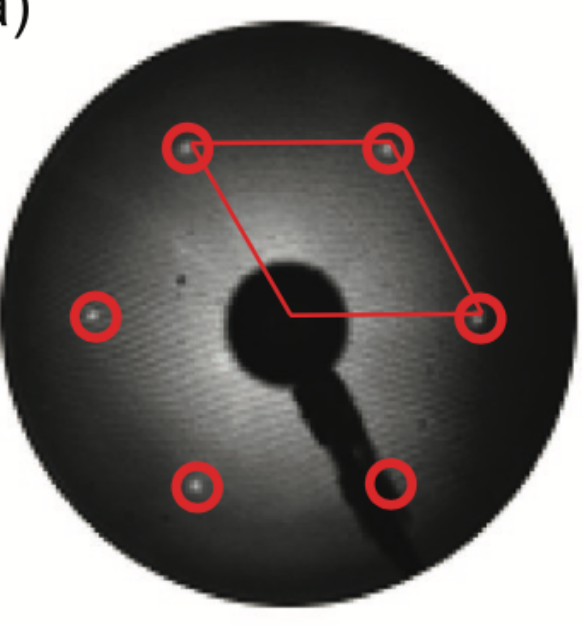

(c)

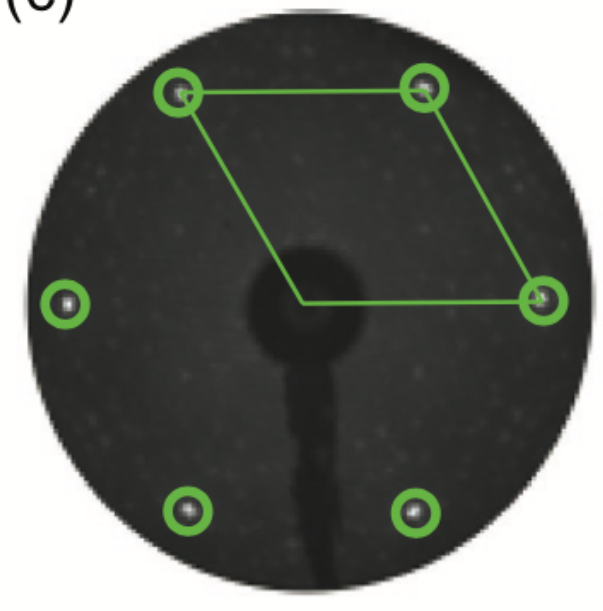

(e)

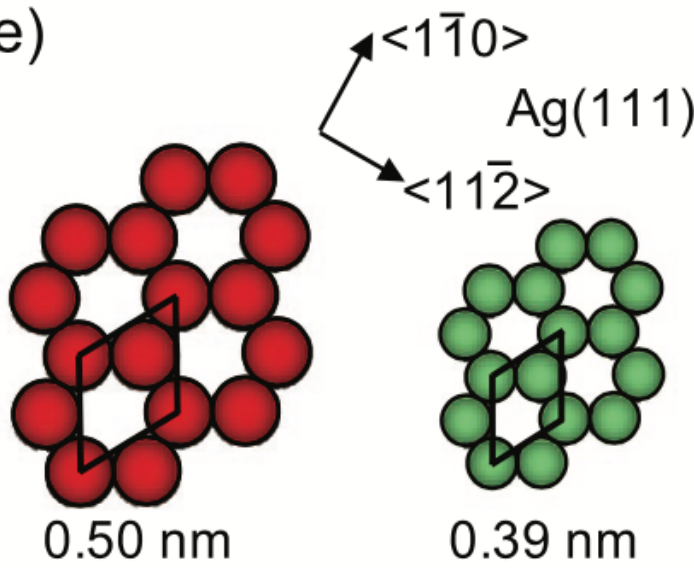

(b)

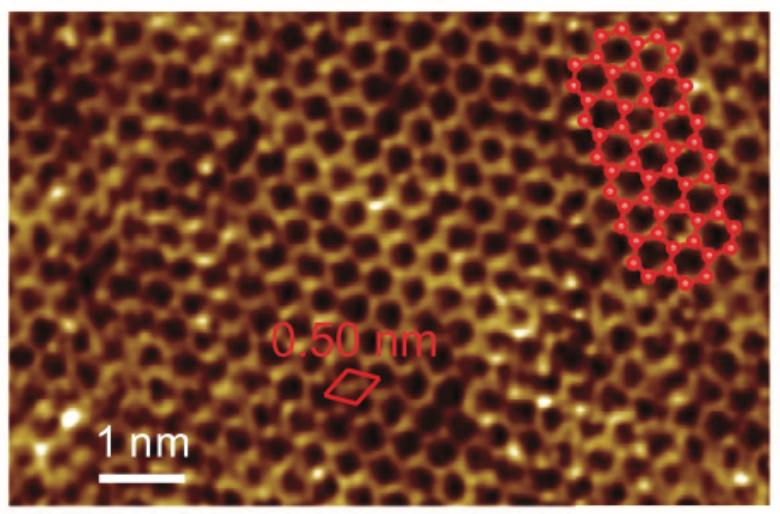

(d)

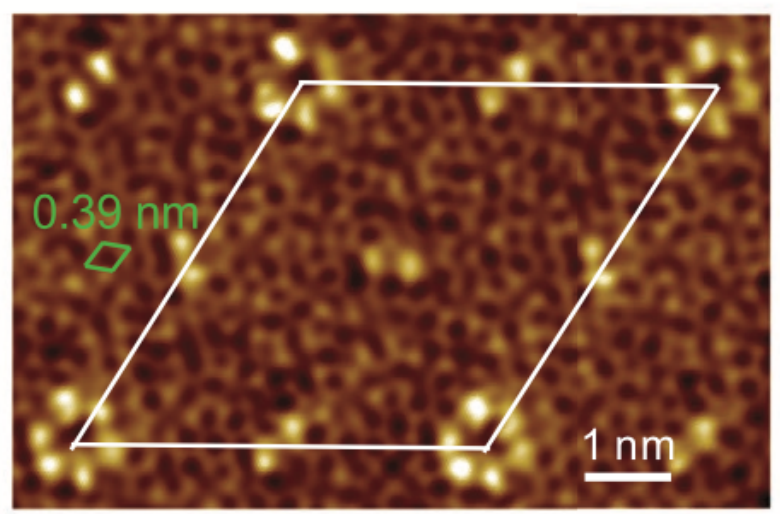

(f)

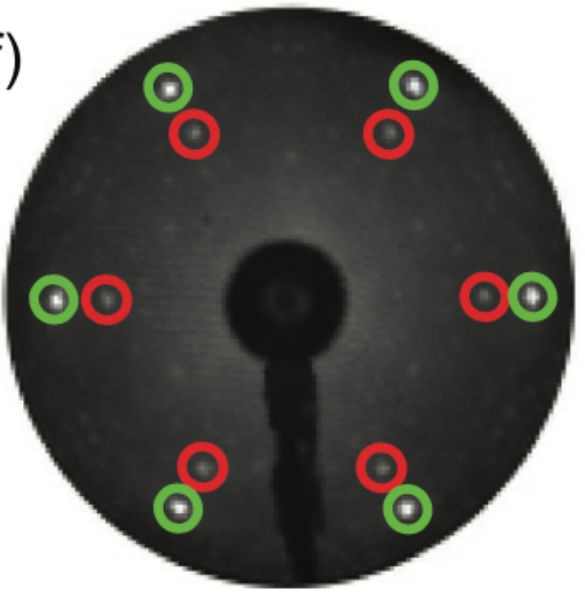

Figure 2. LEED patterns at $36 \mathrm{eV}$ incident electron energy and STM images of a-b) stanene grown by deposition on a $\mathrm{Ag}_{2} \mathrm{Sn}$ surface alloy and $\mathrm{c}-\mathrm{d}$ ) germanene grown by segregation on a $\mathrm{Ag}(111)$ thin film in epi- taxy on a Ge(111) substrate. e) Ball models of the oriented stanene and germanene sheets. f) LEED pattern at $36 \mathrm{eV}$ incident electron energy of the lateral heterostructures of germanene and stanene grown in synergy on a $\mathrm{Ag}(111)$ thin film in epitaxy on a $\mathrm{Ge}(111)$ substrate. 
For direct comparison, Figure $2 f$ shows the LEED pattern for the heterostructure built up on a $\mathrm{Ag}(111)$ thin film grown on a $\mathrm{Ge}(111)$ substrate, following the recipe described above. No other spots, besides those in Figure 2a,c are seen, which indi- cates that no ordered new structure composed of intermixed $\mathrm{Sn}$ and $\mathrm{Ge}$ atoms is formed. Furthermore, within the resolu- tion limits, the positions of the LEED spots are identical to a superposition of those stemming from the sole germanene and stanene overlayers, shown in Figure 2a,c. It indicates that pure germanene and stanene domains are obtained by our methodology, which may possibly point to the realization of lateral heterojunctions.

Actually, this is effectively confirmed in STM micro- scopy observations. Figure 3a shows the STM image corre- sponding to the LEED pattern shown in Figure 2f. On the (111) terraces of the silver thin film, the characteristic $2 \mathrm{D}(3 \sqrt{2} 1 \times 3 \sqrt{2} 1) \mathrm{R} 10.9^{\circ}$ germanene superstructure, matching a $\mathrm{Ag}(111)(7 \sqrt{ } 7 \times 7 \sqrt{ } 7) \mathrm{R} 19^{\circ}$ superperiodicity,[24] is easily recog- nized upon comparison with Figure $2 \mathrm{~d}$. It surrounds, two rounded $2 \mathrm{D}$ islands, with diameters of several tens $\mathrm{nm}$. One is observed on a single terrace, while the other one carpets the straight step. From the section profile along $A-B$, the height difference of the two successive germanene covered terraces is $0.23 \mathrm{~nm}$ (Figure $3 \mathrm{~b}$ ), corresponding to the mono- atomic step height of $\mathrm{Ag}(111)$. The embedded island is only $0.04 \mathrm{~nm}$ higher than the surrounding germanene covered terrace, as determined from the C-D section profile shown in Figure 3c. This is just the difference between the $\mathrm{Sn}$ and $\mathrm{Ge}$ atomic diameters. We stress that no 3D islands are observed in the wide-scale STM images. Furthermore, as the areal ratio of the observed stanene islands is rather smaller than the one which could be expected for the Sn 0.3 monolayer (ML) deposition, therefore, some Sn atoms might have con- currently dissolved into the $\mathrm{Ag}(111)$ thin film.

A zoom-in at atomic-scale of the STM image of the island shown in Figure $3 \mathrm{~d}$ (red square in Figure $3 a$ ) reveals the clear honeycomb structure of epitaxial stanene, matching a $\mathrm{Ag}(111)(\sqrt{3}$ $\times \sqrt{3}) \mathrm{R} 30^{\circ}$ superstructure initially formed by a Ag2 Sn sur- face alloy underneath, as discussed above, and precisely char- acterized earlier.[26] The honeycomb structure appears highly perfect, exhibiting no specific distortion, defects or protrusions that could have originated from substituted or on-top Ge atoms. It means that monolayer stanene is prepared without any replacement or adsorption of $\mathrm{Ge}$ atoms.

Similarly, a zoom-in at atomic-scale of the surrounding germanene area (Figure $3 e$ ) reveals the specific features, namely hexagons and double protrusions constitutive of the 2D $(3 \sqrt{2} 1 \times$ $3 \sqrt{21) R} 10.9^{\circ}$ germanene superstructure, which char- acterize the STM images of the perfectly oriented germanene sheet formed by germanium segregation and ordered assembly on the silver thin film initially grown on $\mathrm{Ge}(111),[24]$ as already displayed in Figure $2 \mathrm{~d}$.

We now underline that if instead of the pure $\mathrm{Ag}(111)$ tem- plate, the Ag2Sn surface alloy with $(\sqrt{3} \times \sqrt{3}) \mathrm{R} 30^{\circ}$ periodicity with respect to $\mathrm{Ag}(111) 1 \times 1$ was lying beneath the germanene sheet, the $(7 \sqrt{ } 7 \times 7 \sqrt{ } 7) \mathrm{R} 19^{\circ}$ superstructure with respect to silver $(111)$ could not have formed, because of the crystallographic incompatibility between $(7 \sqrt{7} \times 7 \sqrt{ } 7) \mathrm{R} 19^{\circ}$ and $(\sqrt{3} \times \sqrt{3}) \mathrm{R} 30^{\circ}$ super periodicities. 
(a)

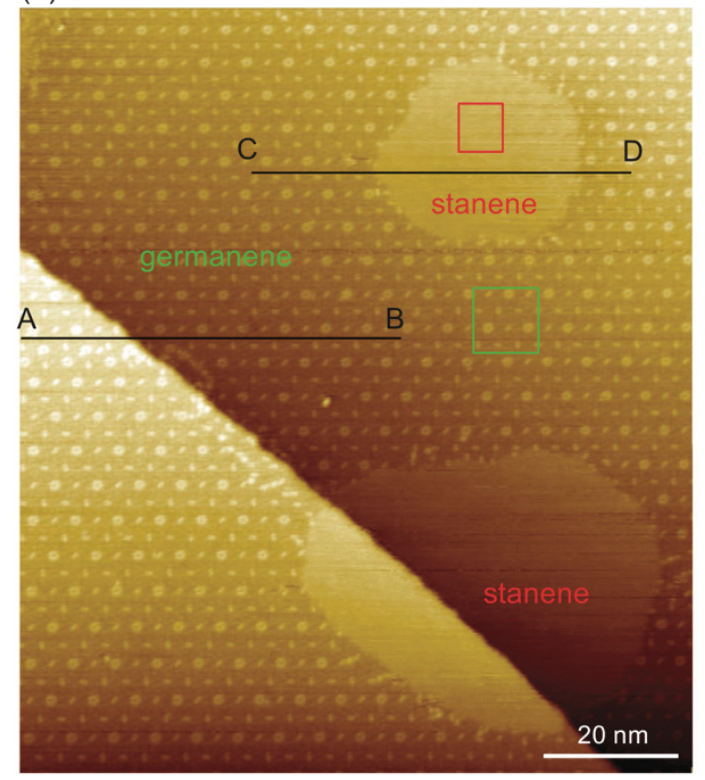

(b)

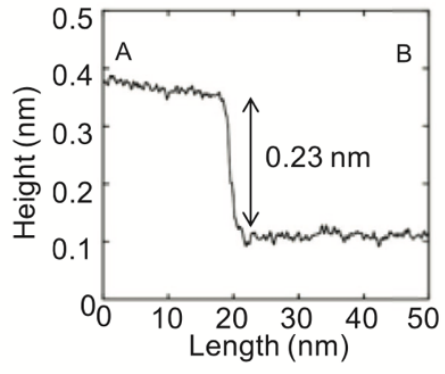

(d)

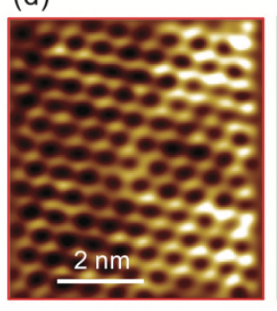

(e) (c)
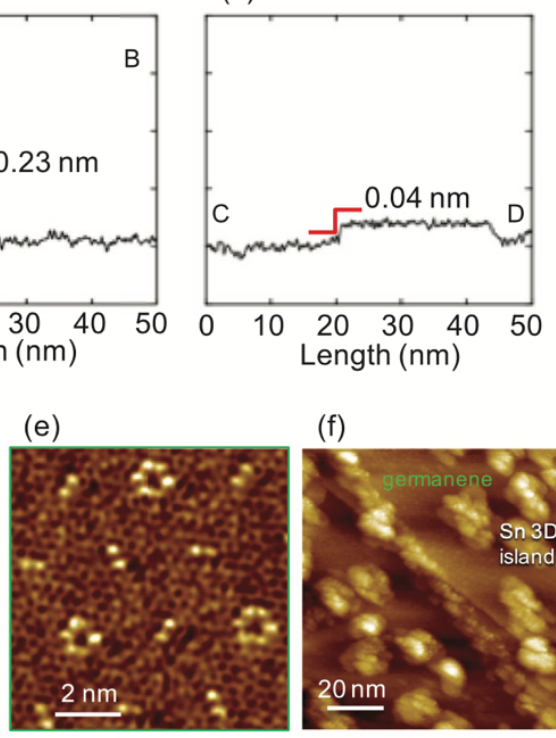

(f)

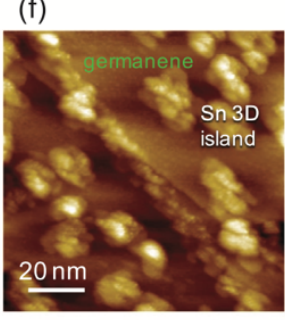

Figure 3. Lateral heterostructures of germanene and stanene observed at RT. They were prepared on a $\mathrm{Ag}(111)$ thin film grown on a $\mathrm{Ge}(111)$ substrate after annealing at the substrate temperature of $500^{\circ} \mathrm{C}$. During the cooling process, where Ge segregation takes place, a Sn deposition of $0.3 \mathrm{ML}$ at $200^{\circ} \mathrm{C}$ was performed. a) Large-scale STM image $\left(U_{S}=\right.$ $+1.5 \mathrm{~V}, I=200 \mathrm{pA}$ ). b) Section profile along the A-B black line in (a). c) Section profile along the C-D black line in (a). d) Atomic-scale STM image of the embedded stanene island within the red square in (a) $\left(U_{S}=+0.4 \mathrm{~V}, I=200 \mathrm{pA}\right)$. e) Atomic- scale STM image of the surrounding germanene sheet within the green square in (a) $\left(U_{S}=+1.0 \mathrm{~V}, I=200 \mathrm{pA}\right)$. f) Wide-scale STM image prepared on a $\mathrm{Ag}(111)$ thin film grown on a $\mathrm{Ge}(111)$ substrate after annealing at the substrate temperature of $500^{\circ} \mathrm{C}$ with a Sn deposition of $0.3 \mathrm{ML}$ at $150^{\circ} \mathrm{C}$ during the cooling process. $\left(U_{S}=+1.5 \mathrm{~V}, I=200 \mathrm{pA}\right)$, showing $\mathrm{Sn} 3 \mathrm{D}$ islanding at this too low temperature.

When the temperature chosen for Sn deposition during the cooling process of the $\mathrm{Ag}(111)$ thin film on the $\mathrm{Ge}(111)$ substrate is higher than about $220^{\circ} \mathrm{C}$, for example $260^{\circ} \mathrm{C}$, the surface is completely covered by germanene and no stanene phase is observed: the STM images look like the one shown in Figure 2d. Furthermore, as no 3D Sn islands appear, all deposited Sn atoms have dissolved into the silver film.

At variance, as shown above in Figure $3 \mathrm{a}, \mathrm{Sn}$ atoms form large atom-thin $2 \mathrm{D}$ stanene islands upon deposition at $200^{\circ} \mathrm{C}$.

We note that most often more or less rounded stanene islands are formed, although elongated ones are also present. This points to rather isotropic on-surface diffusion processes, as well as similar lateral growth velocities along the main crystal- lographic directions, consequently indicating not markedly dif- ferent edge energies.

Now, if instead, the deposition temperature chosen during the cooling process is too low, for example, $150^{\circ} \mathrm{C}$ or less, on the contrary, 3D Sn islands are formed over the germanene sheet, as shown in Figure $3 f$. These results indicate that once a full germanene sheet overlays the 
$\mathrm{Ag}(111)$ surface, $\mathrm{Sn}$ atoms cannot kick away Ge atoms from germanene; instead, they just form 3D Sn islands on top of the germanene overlayer.

Hence, if $\mathrm{Sn}$ is deposited before the germanene sheet fully covers the entire surface upon cooling the substrate, $\mathrm{Sn}$ atoms can form stanene and/or a Ag $2 \mathrm{Sn}$ surface alloy on the remaining bare areas. In other words, the heterostructures can be realized only in a narrow temperature window because of the competitive, kinetically driven processes, namely, on the one hand, $\mathrm{Ge}$ atom diffusion through the thin $\mathrm{Ag}(111)$ film, segregation on top, and surface diffusion to permit 2D germanene nucleation and lateral spreading, and, on the other hand, enough Sn atoms diffusing on the surface to nucleate 2D stanene islands on areas not yet covered by germanene. Said differently, if the Sn deposition temperature is too high, tin dissolves into the Ag thin film, if it is too low, a complete ger- manene overlayer has enough time to form, on top of which tin cannot form a stanene wetting layer, which results in 3D growth. Hence, the temperature chosen for the deposition of tin plays a crucial role.

A close-up STM image of the stanene island of Figure 3a, is shown in Figure 4a to examine the boundaries of the ger- manene and stanene domains of the lateral heterostructures. Red solid lines indicate some preferential directions for the domain boundaries. They are $30^{\circ}$ clockwise rotated toward the $\mathrm{Ag}(111)<112>$ direction, which is the germanene zig-zag direction, and $19^{\circ}$ anti-clock-wise rotated from a unit vector of the $\mathrm{Ag}(111)(7 \sqrt{ } 7 \times 7 \sqrt{ } 7) \mathrm{R} 19^{\circ}$ superstructure. Figure $4 \mathrm{~b}$ displays the boundary region between stanene and germanene (for a different preparation) at atomic resolution. Although it is dif- ficult to image both sides in the same tunneling conditions, one clearly recognizes a zig-zag edge for the stanene island on the left side, and the fingerprint protrusions of the germanene sheet on the right side, also pointing to a zig-zag termination. If not truly atomically perfect, as one could desire, neverthe- less, the interface appears very narrow, with minimal disorder, pointing to a fairly abrupt junction. 
(a)

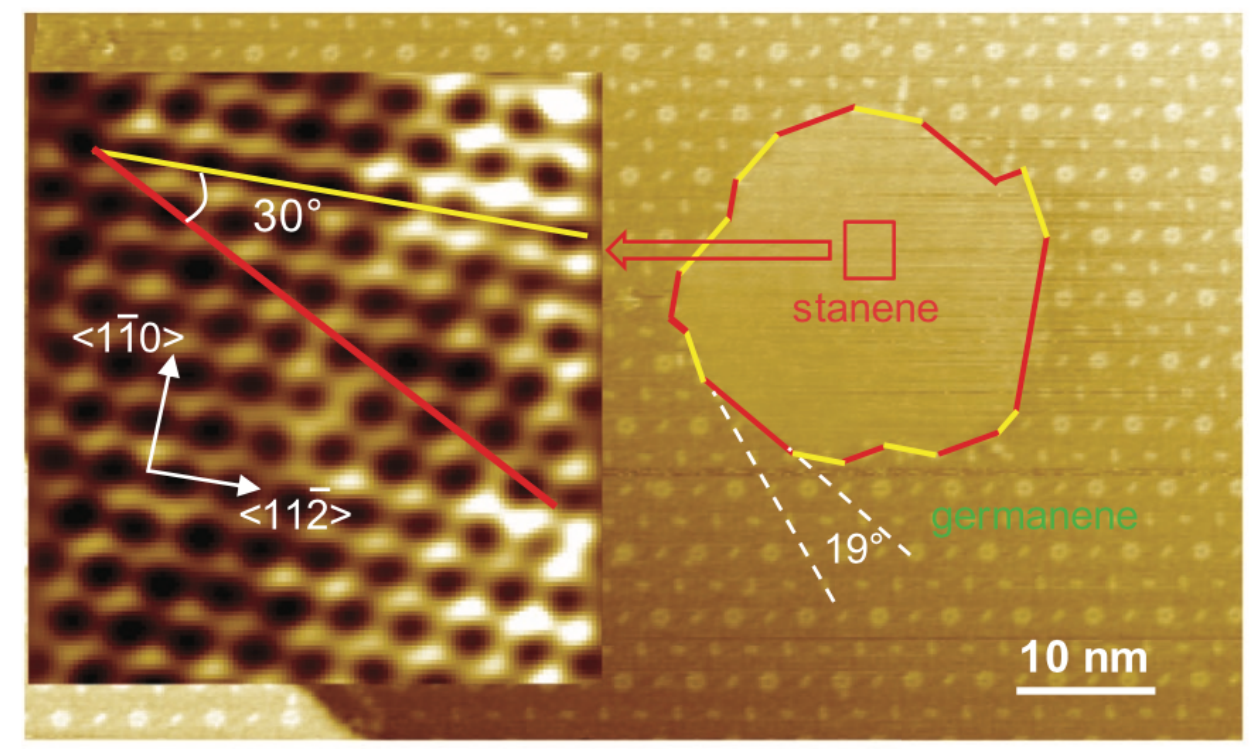

(b)

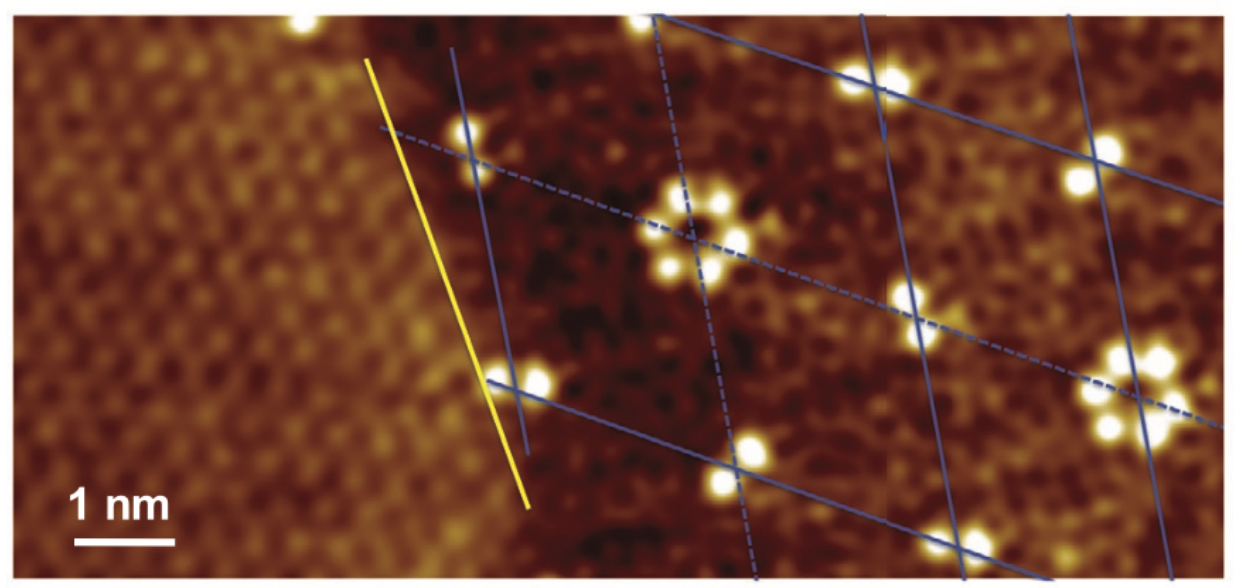

Figure 4. a) STM image of the lateral in-plane heterostructure of stanene and germanene $\left(U_{S}\right.$ $=+1.5 \mathrm{~V}, I=200 \mathrm{pA}$ ). The solid yellow and red lines represent domain boundaries with zigzag and armchair edges. b) High- resolution STM image of a zig-zag boundary of the lateral inplane hetero- structure of germanene and stanene $\left(U_{S}=+1.5 \mathrm{~V}, I=200 \mathrm{pA}\right)$.

A schematic illustration of perfect zig-zag and armchair junc- tions between germanene and stanene on $\mathrm{Ag}(111)$ is displayed in Figure 5. The unit cells of the honeycomb structures of ger- manene and stanene with sides $1.35 \mathrm{aAg}$ and $\sqrt{3} \mathrm{aAg}$ along the $\mathrm{Ag}<112>$ and equivalent directions are rotated by $30^{\circ}$ from that of $\mathrm{Ag}(111)$, whose $\mathrm{AAg}$ sides are along the $\mathrm{Ag}<110>$ and equiva- lent directions.

From the STM image of Figure $4 \mathrm{a}$, the domain boundary of germanene is rotated by $19^{\circ}$ toward the anti-clock-wise direction from the $\mathrm{Ag}(111)(7 \sqrt{ } 7 \times 7 \sqrt{ } 7) \mathrm{R} 19^{\circ}$ superstructures; a germanene armchair edge is thus formed. The STM images also indicate that the domain boundaries for stanene are rotated $30^{\circ}$ toward the clock-wise direction from the $\mathrm{Ag}(111)(\sqrt{3} \times \sqrt{3}) \mathrm{R} 30^{\circ}$ superstructures. Hence, a stanene armchair edge is also formed. Therefore, the domain boundary, 
which is along the $\mathrm{Ag}(111)<1-10>$ direction, has armchair edges for both domains. Because the orientations of the honeycomb structures of germanene and stanene are identical to each other, the domain boundary has the same edge structure, but with different atom spacings. We notice that most domain boundaries share armchair edges, signaling minimum energy equilibrium configuration, but zigzag edges are also observed in the STM images, as shown in Figure 4b.

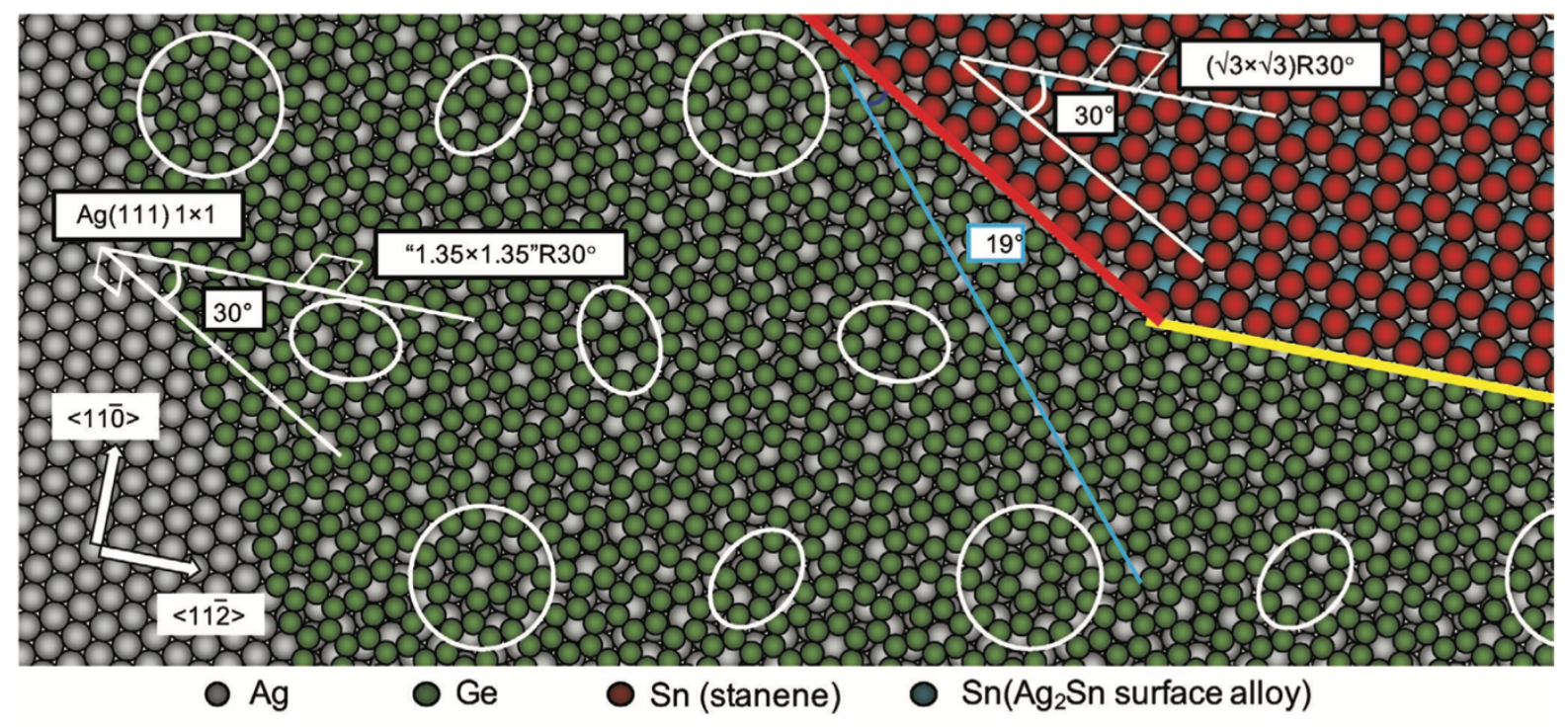

Figure 5. Structural model of the lateral heterostructure of germanene and stanene on $\mathrm{Ag}(111)$ with armchair (marked by a red line) and zig-zag (marked by a yellow line), keeping the unit cell of germanene and stanene, namely, $1.35 \times 1.35 \mathrm{R} 30^{\circ}$ and $(\sqrt{3} \times \sqrt{3}) \mathrm{R} 30^{\circ}$, respectively. The areal positions corresponding to the hexagonal and double-dotted protrusions in Figure $3 e$ are marked by white circles and ovals. 


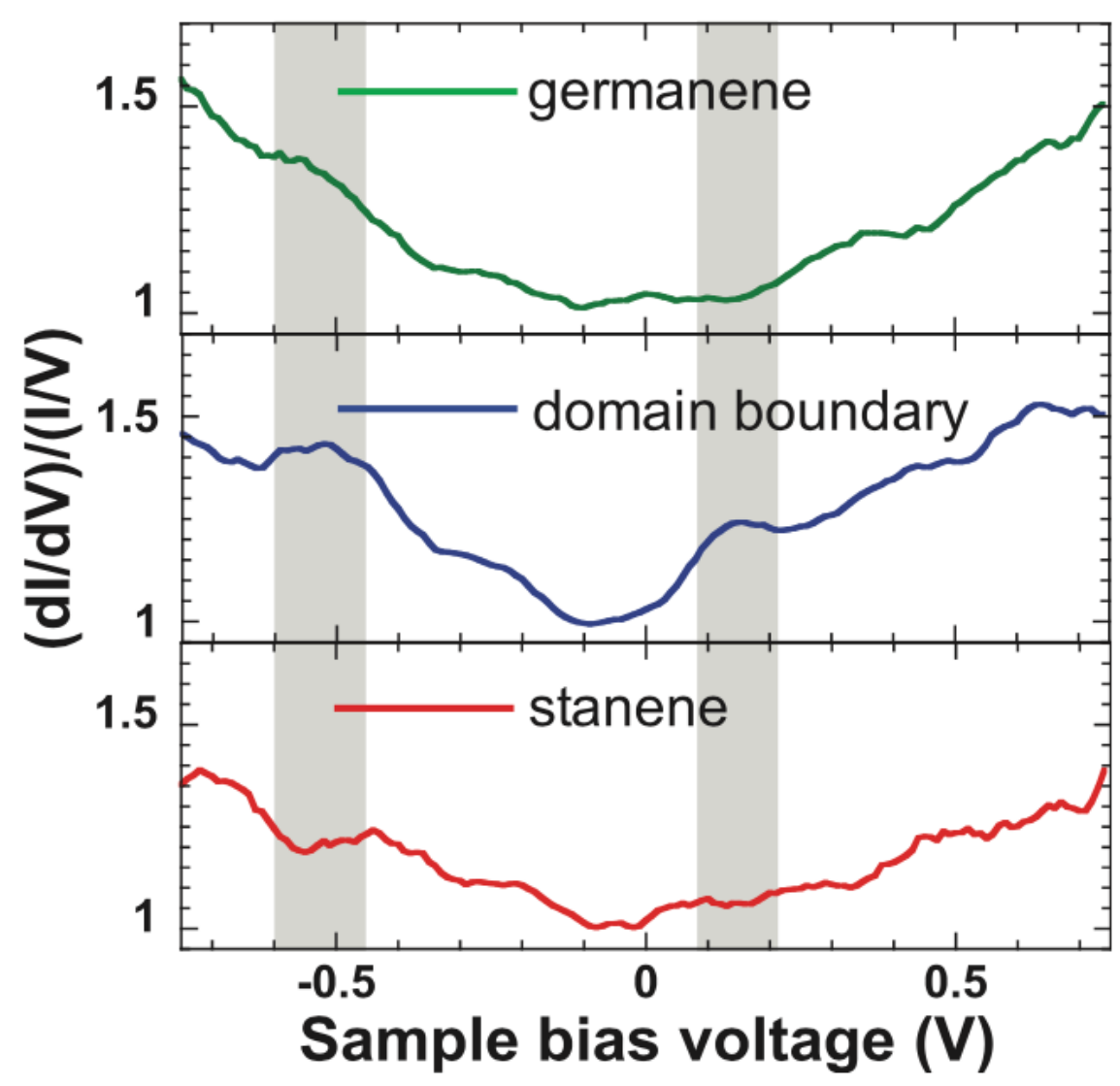

Figure 6. STS spectra of a germanene zone, a zigzag domain boundary region, and a stanene zone of the heterostructure on the same terrace of the $\mathrm{Ag}(111)$ thin film.

Figure 6 shows ( $d / / d V) /(I / V)$ STS spectra for the lateral het- erostructure of stanene embedded in germanene recorded on the germanene and stanene domains, and over a region span- ning a zig-zag domain boundary. Clearly two characteristic peaks emerge at the domain boundary at -0.5 and $+0.15 \mathrm{eV}$ sample biases. We relate these features to a characteristic elec- tron density of states for filled and empty edge states.

The fabrication of the first silicene transistor operating at RT in $2015[30]$ has opened exciting prospects for future 2D electronics.[33,34] Compatible with the current Si-based tech- nology, germanene and stanene, which are 2D topological insu- lators with significantly stronger spinorbit couplings[31] are highly promising new contenders.[34] The realization of a lateral inplane heterostructure between germanene and embedded stanene nanodots is unprecedented. To the best of our knowl- edge, until now, such heterostructures have been obtained, on the one hand, only between graphene and h-BN since 2012[32-34] and with transition metal dichalcogenides since 2014, on the other hand.[8] These novel heterostructures will further boost fundamental studies, not only in electronics, but also, typically, in spintronics and optoelectronics.[37] Progress in engineering, for example, to tailor nanoribbons and lateral superlattices, and use such effects as negative differential resistance, can be [35-38] anticipated. Recently, multilayers of germanene, stanene, and antimonene have been grown directly on semiconducting molybdenum disulfides;[39] indeed, an exciting prospect would be the lateral growth of heterostructures between them. 
To summarize, revolutionary developments can be foreseen, none the least in quantum science, but also for new tantalizing functional nano-devices.

\section{Conclusion}

We have successfully prepared at the nanoscale nearly atomi- cally precise lateral monolayer heterostructures of stanene embedded in germanene on a silver (111) thin film epitaxially grown on a $\mathrm{Ge}(111)$ substrate thanks to a synergetic combina- tion of germanium segregation from the $\mathrm{Ge}(111)$ substrate and tin deposition from a $\mathrm{Sn}$ evaporation source. The combined STM and LEED observations testify that the lateral structures on either side of the heterojunctions are pure germanene and pure stanene monolayers, respectively. The domain boundaries have mostly armchair edges, but zigzag edges are also formed. From the STS spectra, 1D edge states are revealed. To the best of our knowledge this is the first realization of a lateral hetero- junction between group 14 elemental post-graphene 2D mate- rials, whose outstanding electronic properties open exciting prospects for disruptive technologies, such as the future fabri- cation of functional 2D nano-devices compatible with conven- tional Si-based technology.

\section{Experimental Section}

The experiments were performed using an ultrahigh-vacuum (UHV) system at Nagoya University. The system consisted of a preparation chamber with a base pressure below $5 \times$ $10^{-10} \mathrm{mbar}$, and an analysis chamber with a base pressure below $10^{-10} \mathrm{mbar}$.

Silver thin films (100 nm thick) were prepared on a Ge(111) substrate by conventional thermal evaporation. The details of the sample preparation of $\mathrm{Ag}$ thin films on $\mathrm{Ge}(111)$ and segregation phenomena of the $\mathrm{Ge}$ atoms by subsequent annealing under $\mathrm{N}_{2}$ atmosphere are described in previous reports. [40-42] Clean $\mathrm{Ag}$ (111) surfaces were prepared by $2 \mathrm{keV} \mathrm{Ar}^{+}$ion sputtering at RT, followed by annealing the sample at the temperature of $400{ }^{\circ} \mathrm{C}$. Annealing was performed by electron bombardment of the sample holder at an emission current of $5 \mathrm{~mA}$ and a voltage of $-650 \mathrm{~V}$. The sample temperature was monitored with a radiation thermometer and a type $\mathrm{K}$ thermocouple mounted on the base plate of the sample holder. The cleanliness of the surface was measured by Auger electron spectroscopy after sputtering and annealing; no contaminants, such as $\mathrm{C}$ and $\mathrm{O}$, were observed within the detection limits, and a sharp $(1 \times 1)$ LEED pattern was observed.

After annealing the sample at $500^{\circ} \mathrm{C}$, tin was deposited onto the $\mathrm{Ag}(111)$ surface during the cooling process at various temperatures from $300^{\circ} \mathrm{C}$ down to RT in UHV from a well degassed quartz crucible evaporator operating at a deposition rate of approximately $0.1 \mathrm{ML} \mathrm{min}^{-1}$, as measured by placing a quartz crystal microbalance at the sample position. $1 \mathrm{ML}$ was defined as a $\mathrm{Sn}$ overlayer with the atomic density of a $\mathrm{Ag}(111)$ plane: $1.38 \times 10^{15}$ atoms $\mathrm{cm}^{-2}$. The deposition rate was accurately calibrated in advance by Rutherford backscattering spectroscopy (RBS). For the RBS measurement, a graphite substrate with an ultra-thin tin film on the surface was prepared. Details of the experimental setup for RBS have been published elsewhere.[43,44] The amounts of deposited tin were about $0.3 \mathrm{ML}$; errors in mentioned coverage ratios were less than $5 \%$.

The system was equipped with a rear-view LEED system operating with a LaB 6 filament and a UHV STM system (Omicron STM-1). All STM images presented were acquired at RT with W tips, electrochemically etched in a $\mathrm{KOH}$ solution, in a constant current mode. All apparatuses were situated on an air damper with an active vibration isolation system (Kurashiki). STS 
spectra were obtained together with the simultaneous STM measurements by numerical derivative of tunneling current $(I)$ versus sample bias voltage $(V)$ and averaging over 20 equivalent sites to reduce noise.

\section{Acknowledgements}

J.Y. acknowledges financial support from Toyoaki Scholarship Foundation and the Murata Science Foundation in 2017 and 2019, respectively. G.L.L. acknowledges support from Nagoya University thanks to two "Eminent Foreign Scientist" Invitation Awards in 2015-2016 and in 2019-2020, as well as an Invitational Fellowship for Research in Japan by the Japan Society for the Promotion of Science (JSPS) in 2017.

\section{References}

[1] P. Vogt, P. de Padova, C. Quaresima, J. Avila, E. Frantzeskakis, M. C. Asensio, A. Resta, B. Ealet, G. Le Lay, Phys. Rev. Lett. 2012, 108, 155501.

[2] A. Fleurence, R. Friedlein, T. Ozaki, H. Kawai, Y. Wang, Y. Yamada-Takamura, Phys. Rev. Lett. 2012, 108, 245501.

[3] M. E. Dávila, L. Xian, S. Cahangirov, A. Rubio, G. Le Lay, New J. Phys. 2014, 16, 095002.

[4] F. Zhu, W. Chen, Y. Xu, C. Gao, D. Guan, C. Liu, D. Qian, S.-C. Zhang, J. Jia, Nat. Mater. 2015, 14, 1020.

[5] J. Yuhara, B. He, N. Matsunami, M. Nakatake, G. Le Lay, Adv. Mater. 2019, 31, 1901017.

[6] L. Liu, J. Park, D. A. Siegel, K. F. McCarty, K. W. Clark, W. Deng, L. Basile, J. C. Idrobo, A.-P. Li, G. Gu, Science 2014, 343, 163.

[7] J. Li, V. B. Shenoy, Appl. Phys. Lett. 2011, 98, 013105.

[8] J. Zhao, K. Cheng, N. Han, J. Zhang, Wiley Interdiscip. Rev.: Comput. Mol. Sci. 2018, 8, e1353.

[9] X. Liu, M. C. Hersam, Sci. Adv. 2019, 5, eaax6444.

[10] X.-S. Ye, Z.-G. Shao, H. Zhao, L. Yang, C.-L. Wang, RSC Adv. 2014, 4, 21216.

[11] L. Matthes, O. Pulci, F. Bechstedt, J. Phys.: Condens. Matter 2013, 25, 395305.

[12] A. Debernardi, L. Marchetti, Phys. Rev. B 2016, 93, 245426.

[13] S. Kaneko, H. Tsuchiya, Y. Kamakura, N. Mori, M. Ogawa, Appl.

Phys. Express 2014, 7, 035102.

[14] L. Meng, Y. Wang, L. Zhang, S. Du, R. Wu, L. Li, Y. Zhang, G. Li, 
H. Zhou, W. A. Hofer, H.-J. Gao, Nano Lett. 2013, 13, 685.

[15] L. Li, S. Lu, J. Pan, Z. Qin, Y. Wang, Y. Wang, G. Cao, S. Du,

H.-J. Gao, Adv. Mater. 2014, 26, 4820.

[16] M. Derivaz, D. Dentel, R. Stephan, M.-C. Hanf, A. Mehdaoui,

P. Sonnet, C. Pirri, Nano Lett. 2015, 15, 2510.

[17] J. Gou, Q. Zhong, S. Sheng, W. Li, P. Cheng, H. Li, L. Chen, K. Wu, 2D Mater. 2016, 3, 045005.

[18] Z. Qin, J. Pan, S. Lu, Y. Shao, Y. Wang, S. Du, H.-J. Gao, G. Cao, Adv. Mater. 2017, 29, 1606046.

[19] C.-H. Lin, A. Huang, W. W. Pai, W.-C. Chen, T.-Y. Chen, T.-R. Chang,

R. Yukawa, C.-M. Cheng, C.-Y. Mou, I. Matsuda, T.-C. Chiang,

H.-T. Jeng, S.-J. Tang, Phys. Rev. Mater. 2018, 2, 024003.

[20] P. Bampoulis, L. Zhang, A. Safaei, R. van Gastel, B. Poelsema,

H. J. W. Zandvliet, J. Phys.: Condens. Matter 2014, 26, 442001.

[21] L. Zhang, P. Bampoulis, A. N. Rudenko, Q. Yao, A. van Houselt, B. Poelsema, M. I. Katsnelson, H. J. W. Zandvliet, Phys. Rev. Lett.

$2016,116,256804$

[22] L. Persichetti, F. Jardali, H. Vach, A. Sgarlata, I. Berbezier,

M. De Crescenzi, A. Balzarotti, J. Phys. Chem. Lett. 2016, 7, 3246.

[23] J. Zhuang, C. Liu, Z. Zhou, G. Casillas, H. Feng, X. Xu, J. Wang, W. Hao, X. Wang, S. X. Dou, Z. Hu, Y. Du, Adv. Sci. 2018, 5, 1800207.

[24] J. Yuhara, H. Shimazu, K. Ito, A. Ohta, M. Araidai, M. Kurosawa, M. Nakatake, G. Le Lay, ACS Nano 2018, 12, 11632.

[25] J. Gou, L. Kong, H. Li, Q. Zhong, W. Li, P. Cheng, L. Chen, K. Wu, Phys. Rev. Mater. 2017, 1, 054004.

[26] J. Yuhara, Y. Fujii, K. Nishino, N. Isobe, M. Nakatake, L. Xian, A. Rubio, G. Le Lay, 2D Mater. 2018, 5, 025002.

[27] J. Deng, B. Xia, X. Ma, H. Chen, H. Shan, X. Zhai, B. Li, A. Zhao, Y. Xu, W. Duan, S.-C. Zhang, B. Wang, J. G. Hou, Nat. Mater. 2018, 17, 1081. 
[28] R. W. Olesinski, G. J. Abbaschian, J. Alloy Phase Diagrams 1984, 5, 265. [29] R. W. Olesinski, G. J. Abbaschian, J. Alloy Phase Diagrams 1984,

$5,180$.

[30] L. Tao, E. Cinquanta, D. Chiappe, C. Grazianetti, M. Fanciulli,

M. Dubey, A. Molle, D. Akinwande, Nat. Nanotechnol. 2015,

$10,227$.

[31] M. Kurpas, P. E. Faria Junior, M. Gmitra, J. Fabian, Phys. Rev. B 2019, 100, 125422.

[32] T. H. Nguyen, D. Perilli, M. Cattelan, H. Liu, F. Sedona, N. A. Fox, C. di Valentin, S. Agnoli, Nano Res. 2019, 12, 675.

[33] P. Zhuang, W. Lin, H. Chou, A. Roy, W. Cai, S. K. Banerjee, Nanotechnology 2019, 30, 03LT01.

[34] Q. Wu, W. Wongwiriyapan, J.-H. Park, S. Park, S. J. Jung, T. Jeong,

S. Lee, Y. H. Lee, Y. J. Song, Curr. Appl. Phys. 2016, 16, 1175.

[35] J. Wang, Z. Li, H. Chen, G. Deng, X. Niu, Nano-Micro Lett. 2019, $11,48$.

[36] Y. An, M. Zhang, D. Wu, T. Wang, Z. Jiao, C. Xia, Z. Fu, K. Wang, Phys. Chem. Chem. Phys. 2016, 18, 27976.

[37] M. Ge, C. Si, Carbon 2018, 136, 286.

[38] M. P. Levendorf, C.-J. Kim, L. Brown, P. Y. Huang, R. W. Havener, D. A. Muller, J. Park, Nature 2012, 488, 627.

[39] K.-C. Chen, L.-M. Lee, H.-A. Chen, H. Sun, C.-L. Wu, H.-A. Chen, K.-B. Lin, Y.-C. Tseng, C.-C. Kuan, C.-W. Pao, S.-Y. lin, Semicond. Sci. Technol. 2019, 34, 105020.

[40] M. Kurosawa, A. Ohta, M. Araidai, S. Zaima, Jpn. J. Appl. Phys. 2016, 55,08 NB07. 
[41] K. Ito, A. Ohta, M. Kurosawa, M. Araidai, M. Ikeda, K. Makihara,

S. Miyazaki, Jpn. J. Appl. Phys. 2018, 57, 04FJ05.

[42] K. Ito, A. Ohta, M. Kurosawa, M. Araidai, M. Ikeda, K. Makihara,

S. Miyazaki, Jpn. J. Appl. Phys. 2018, 57, 06 HD08.

[43] J. Yuhara, M. Inoue, K. Morita, J. Vac. Sci. Technol., A 1992 ,

$10,334$.

[44] J. Yuhara, K. Morita, J. Falta, B. H. Müller, M. H.-v. Hoegen, Surf.

Interface Anal. 2001, 31, 754. 